\title{
22. THE RELATION BETWEEN ORBITS AND PHYSICAL CHARACTERISTICS OF METEORS
}

\author{
Ľ. KRESÁK \\ (Astronomical Institute of the Slovak Academy of Sciences, \\ Bratislava, Czechoslovakia)
}

\begin{abstract}
A BST RACT
The relation of physical characteristics of meteors to their orbital elements is investigated using Harvard Super-Schmidt data. A set of characteristic indices is defined, allowing for the effects of geocentric velocity, angle of incidence, magnitude and mass, wherever a correction appears appropriate according to the correlations found by Jacchia et al. (1967). The medians for representative meteor samples are plotted in the semi-major axis/eccentricity diagram and the distribution of each parameter is derived. Although the differences are moderate compared to the measuring errors, six regions of different nature can be distinguished.

The existence of two families of asteroidal meteors is indicated, one of them brought to crossing with the Earth's orbit by drag effects and the other by collision effects in the main asteroid belt. These meteors are characterized by low and uniform beginning heights, high fragmentation, low ablation, low deceleration, and bright wakes. A direct counterpart to this is represented by meteors moving in short-period orbits of higher eccentricity and shorter perihelion distance, which bear resemblance to the long-period and retrograde cometary meteors. Meteors with perihelion distances of less than $0.15 \mathrm{AU}$ tend to resemble the bona fide asteroidal meteors by a progressive increase of fragmentation and decrease of reduced beginning heights and decelerations as the perihelion approaches the Sun. This is attributed to the selective destruction effects of solar radiation.

With the exception of the Draconids, the mean characteristics of meteor showers agree well with those of sporadic meteors moving in similar orbits. It is suggested that the Draconid stream includes a broad variety of meteoric material and that the two peculiar Super-Schmidt meteors on record represent only the less resistive, short-lived component which has already been eliminated from the other showers.
\end{abstract}

\section{Introduction}

The orbital diagram of semi-major axis versus eccentricity proves very suitable for investigating the evolutionary associations among different types of solid interplanetary matter. Its main advantage is that it allows one to represent the virtual distribution of the orbits of comets, asteroids and meteoroids in a uniform manner, and to delineate the potential evolutionary paths expected for different perturbing effects. In a previous paper (Kresák, 1967) some results concerning the origin and evolution of meteor particles have been derived using the statistics of their orbital elements. This was done with due consideration of the selection effects on the observed distribution of the orbits of the comets, asteroids, and meteors. The purpose of the present paper is to check whether there exist significant differences in the physical 
characteristics of meteor families for which different origins and evolutions are suggested.

A general summary of the problem and the results obtained earlier are shown in Figure 1. The orbits of the meteors which will be used for the analysis (Jacchia et al., 1965) are shown by dots; they obviously occupy only the area delimited by the heavy envelope inside which

$$
a(1-e)<1<a(1+e)
$$

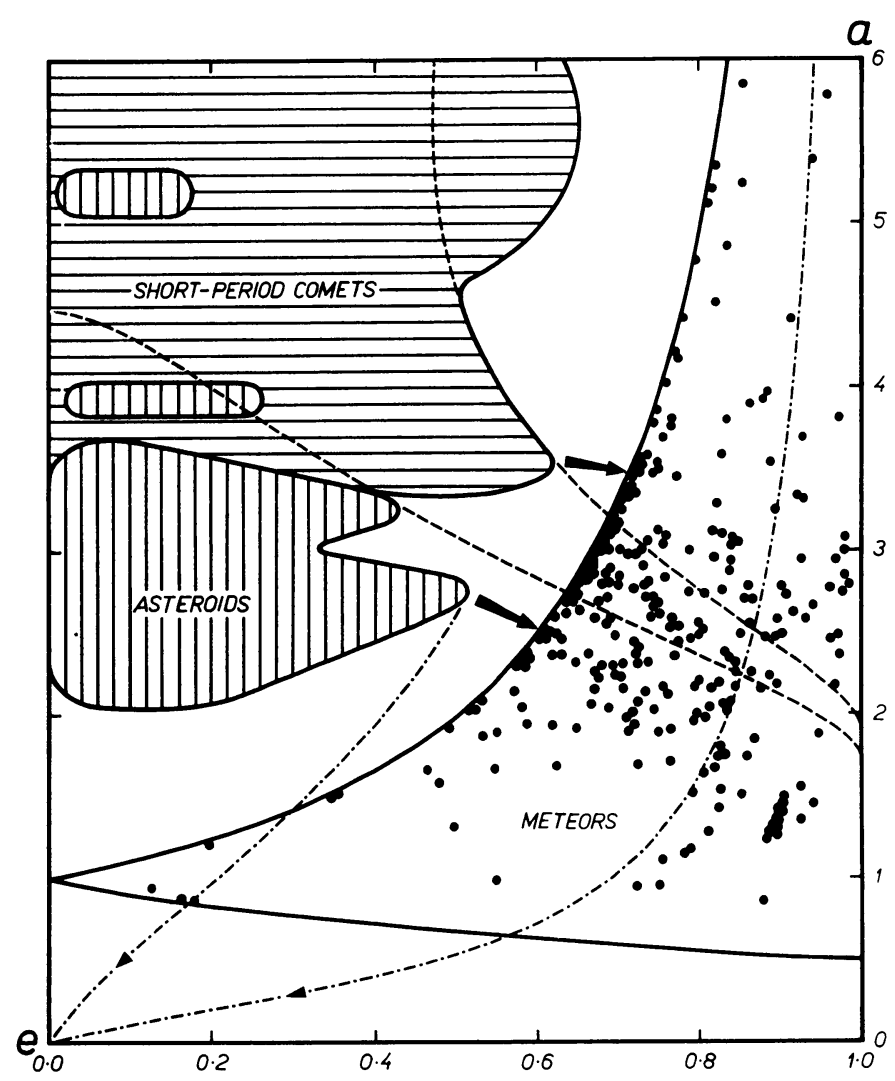

FIG. 1. The distribution of the orbits of meteors, comets and asteroids, and the evolutionary paths in semi-major axis and eccentricity. For explanation see text.

The main concentrations of the orbits of comets and asteroids are situated far outside this region. They are schematically represented by the shaded areas whose outlines roughly correspond to a uniform relative abundance of orbits. Other isopleths can be found in the original paper (Kresák, 1967) from which the direction of the minimum gradients for the short-period comets and asteroids, indicated by the two 
arrows, are taken. Around the points where the arrows meet the envelope of the meteor region (i.e. the region of encounter with the Earth), we should expect the highest concentration of short-period cometary and asteroidal orbits, respectively. These are the densest regions of stray bodies on the outskirts of the two systems and, in the case of the asteroids, also the region most effectively fed by the products of mutual collisions in the main asteroid belt.

Gravitational perturbations tend to keep the aphelia of the orbits resulting from the disintegration of short-period comets near the orbit of Jupiter, with a constant value of the Tisserand invariant for the system Sun-Jupiter-Meteor. Assuming a zero inclination, we get for the core of the system of short-period comets of Jupiter's family the condition

$$
0.53<a^{-1}+2 a_{\mathrm{J}}^{-3 / 2} a^{1 / 2}\left(1-e^{2}\right)^{1 / 2}<0 \cdot 58 .
$$

The region delineated by the two dashed curves defined by (2) constitutes a natural continuation of the short-period comet region. Displacements in both directions along the lines are equally probable but the funnel-like shape of the area is favourable for concentrating the orbits in the region of encounter with the Earth. For low inclinations, characteristic of short-period comets, this region extends upwards to Jupiter's perturbational barrier.

The drag effects, such as the Poynting-Robertson effect, tend to displace the orbits down and to the left. Two of such evolutionary paths, for which the drag constant is

$$
a e^{-4 / 5}\left(1-e^{2}\right)=3.40 \text { and } a e^{-4 / 5}\left(1-e^{2}\right)=0 \cdot 70 \text {, }
$$

are indicated by dash-dotted lines.

The former value gives a tentative boundary of transformed asteroidal orbits during their spiralling from the main belt to the Sun; the latter is a characteristic example of the evolutionary path of a long-period cometary orbit, with $q=0.35$.

It may be noted that the asteroidal meteors, if present, may concentrate in two separate areas: the region of collisional displacement (around $a=2 \cdot 5, e=0 \cdot 6$ ) and the region of displacement by drag effects (around $a=1 \cdot 0, e=0 \cdot 2$ ). Indications of such a double concentration have already been found in the analysis of the distribution of meteor orbits (Kresák, 1967).

\section{Observational Data}

The data to be used for the present study were taken from the detailed analysis of 413 high-precision Super-Schmidt meteor photographs (Jacchia and Whipple, 1961) made by Jacchia et al. (1967). Under investigation were three independent characteristics of meteor trails - the presence of wakes, of blending, and the color index - and three derived quantities - the progressive fragmentation, ablation and deceleration indices. The beginning height index, the significance of which has been pointed out by Ceplecha (1967), was added as the seventh quantity, making use of the complete 
lower-precision Super-Schmidt data by McCrosky and Posen (1961). An attempt was made to eliminate the systematic influence of such factors as the meteor's no-atmosphere velocity, penetration angle, magnitude and mass, using the correlations found by Jacchia, Verniani and Briggs, but only significant correlations were taken into account. Seven types of reduced indices were determined and the median values of each of them were computed for samples of about 10 meteors each, characterized by different combinations of $a, e$ and $i$, i.e. by different positions in the diagram. Meteor showers were treated as separate meteor samples centered at the respective position of the mean orbit. Almost without exception it was found that their characteristics agree well with those of their sporadic surrounding, and therefore they were eventually incorporated into the sporadic meteor distribution. For low inclinations $\left(i<45^{\circ}\right)$, where the data were comprehensive enough, the smoothed distribution of each median index was displayed in an a/e diagram, together with the distribution of extreme individual cases (Figures 2-8). The intercomparison of these diagrams made it possible to delineate tentatively six regions of orbits with significantly different physical characteristics of the meteors. The boundaries of the regions A-E are drawn in dashed lines in each figure. Regions are defined as follows: $i>45^{\circ}$ for $\mathrm{F}, i<45^{\circ}$ for $\mathrm{A}-\mathrm{E}$; $q^{\prime}=a(1+e)>6.5$ for $\mathrm{E}, q^{\prime}<6.5$ for A-D; $q=a(1-e)<0.15$ for $\mathrm{D}, q>0.15$ for A-C. The boundaries between $\mathrm{A}, \mathrm{B}$ and $\mathrm{C}$ are fixed to run along constant values $e=0.50$, $e=0 \cdot 75, a=2 \cdot 20$ and circle arcs centered at $a=2 \cdot 50, e=0 \cdot 60$, and $a=2 \cdot 00, e=1 \cdot 00$. It may be noted that region $A$ consists of two separate areas, corresponding to the two asteroidal regions mentioned above $\left(A_{1}=\right.$ Poynting-Robertson drop from the main belt, $A_{2}=$ collisional and perturbational fringe of the main belt). Region $E$, together with the predominant part of region $\mathrm{F}$, corresponds to long-period orbits with aphelia at least 1 to $1.5 \mathrm{AU}$ beyond the orbit of Jupiter, for which significant differences in physical characteristics have already been found by Jacchia (1955). Region D includes meteors with the strongest effects of heating at perihelion passages less than 0.15 AU from the Sun. There is also a clear relation to Ceplecha's classification of meteors (1967): regions A and B roughly correspond to Ceplecha's type A, region $C$ to Ceplecha's type $C 1$, region $D$ to Ceplecha's type $B$, and regions $E$ and $F$ to Ceplecha's type C2.

Abundance histograms for each characteristic index $S$, constructed separately for each of the six regions, are given at the right-hand side of Figures 2-8. Where the extreme cases, quite exceptionally, fell beyond the limits of the histogram, they were added to the respective last column.

Tables 1 and 2 list the median values of different orbital elements, no-atmosphere velocity, mass (Verniani's scale, 1964), and characteristic indices for each region A-F; also for meteor streams sufficiently represented in the data, and for three types of peculiar meteor trails: those with an abrupt beginning of the light curve $(a)$, those limited to a short flare $(s)$, and those with recorded fracture into two or more pieces $(f) . N$ denotes the number of meteors of the given group. 


\section{Table 1}

\begin{tabular}{|c|c|c|c|c|c|c|c|c|c|c|}
\hline & $N$ & $\bar{a}$ & $\bar{e}$ & $\bar{q}$ & $i$ & $\bar{v}_{x}$ & $\bar{m}_{\infty}$ & $P_{a}$ & $P_{\delta}$ & $P_{f}$ \\
\hline $\mathbf{A}_{1}$ & 9 & 1.53 & $0 \cdot 35$ & 0.99 & 9 & $14 \cdot 6$ & $1 \cdot 34$ & 11 & 11 & 0 \\
\hline $\mathbf{A}_{2}$ & 15 & $2 \cdot 46$ & 0.61 & 0.96 & 10 & $16 \cdot 9$ & $1 \cdot 32$ & 20 & 0 & 7 \\
\hline B & 127 & 2.77 & 0.70 & 0.82 & 10 & $22 \cdot 0$ & 0.54 & 8 & 3 & 6 \\
\hline C & 65 & $2 \cdot 18$ & 0.83 & 0.37 & 6 & $30 \cdot 4$ & $0 \cdot 19$ & 8 & 2 & 0 \\
\hline D & 38 & $1 \cdot 41$ & 0.90 & 0.14 & 24 & $36 \cdot 7$ & 0.12 & 3 & 3 & 0 \\
\hline E & 29 & 5.84 & 0.90 & 0.57 & 16 & $26 \cdot 7$ & 0.47 & 0 & 3 & 10 \\
\hline $\mathbf{F}$ & 130 & 17.52 & 0.96 & 0.72 & 111 & $58 \cdot 7$ & 0.06 & 4 & 4 & 0 \\
\hline Dra (B) & 2 & 3.33 & 0.70 & 1.00 & 25 & $20 \cdot 2$ & 2.00 & 0 & 0 & 0 \\
\hline S Tau (C) & 13 & 1.88 & 0.82 & 0.34 & 6 & $29 \cdot 3$ & 0.29 & 0 & 0 & 0 \\
\hline N Tau (C) & 6 & $2 \cdot 26$ & 0.85 & 0.33 & 3 & $30 \cdot 9$ & 0.77 & 17 & 0 & 0 \\
\hline a Cap (B-C) & 11 & 2.54 & 0.77 & 0.59 & 7 & $24 \cdot 9$ & 0.20 & 0 & 0 & 0 \\
\hline$\delta$ Aqr (D) & 11 & $2 \cdot 80$ & 0.97 & 0.07 & 26 & $41 \cdot 8$ & 0.06 & 9 & 9 & 0 \\
\hline Gem (D) & 20 & $1 \cdot 37$ & 0.90 & $0 \cdot 14$ & 23 & $36 \cdot 2$ & $0 \cdot 19$ & 0 & 0 & 0 \\
\hline Qua (F) & 8 & 3.06 & $0 \cdot 68$ & 0.97 & 72 & $43 \cdot 1$ & 0.06 & 0 & 0 & 0 \\
\hline $\operatorname{Per}(F)$ & 10 & $22 \cdot 72$ & 0.96 & 0.95 & 113 & $60 \cdot 3$ & 0.11 & 0 & 0 & 0 \\
\hline Ori (F) & 5 & 14.98 & 0.96 & 0.61 & 164 & $67 \cdot 5$ & 0.04 & 60 & 0 & 0 \\
\hline$a$ & 26 & $2 \cdot 42$ & 0.73 & 0.66 & 15 & $24 \cdot 0$ & 0.40 & 100 & 0 & 0 \\
\hline$s$ & 12 & 3.07 & 0.95 & $0 \cdot 16$ & 32 & $38 \cdot 2$ & 0.20 & 0 & 100 & 0 \\
\hline$f$ & 11 & 3.02 & 0.70 & 0.89 & 13 & $20 \cdot 3$ & $5 \cdot 50$ & 0 & 0 & 100 \\
\hline
\end{tabular}

\section{Physical Characteristics of Meteors}

\subsection{WAKES}

The brightness of the wakes is given by Jacchia et al. (1967 - further referred to as JVB) on an arbitrary scale of 0 to 4 , with a subdivision into thirds of the unit interval. The visibility of a wake between the shutter segments essentially depends on the meteor's brightness, and is therefore closely correlated with such quantities as meteor mass or magnitude (JVB, Tables 20.4-20.6). Since the brightness of the wake relative to the head of the meteor was of interest, it was found most reasonable to correct the scale for the effect of the difference $\Delta M$ between the plate limit and the maximum absolute magnitude of the meteor. Besides, this is just the quantity showing the strongest correlation. Since the scale of $W$ was arbitrary, the form of the dependence is unknown, but the following simple relation yields a good fit with observation:

$$
S_{\mathrm{w}}=W-(\Delta M)^{2} / 15-(\Delta M)^{3} / 300 \text {. }
$$

We shall denote $S_{\mathrm{W}}$ as the wake index; the correction to $W$ is -0.5 for $\Delta M=2 \cdot 6$, -1.0 for $\Delta M=3.6,-2.0$ for $\Delta M=4.9$, etc. A negative wake index $S_{\mathrm{w}}$ means that no wake or a very faint wake was recorded; a higher negative value indicates that this was the case in spite of favourable conditions for wake detection (bright meteor). Figure 2 demonstrates that wakes are essentially absent in region $C$, while they occur frequently in region $B$ and, in particular, in $A$. Most conspicuous are wakes in region $A_{2}$ where 


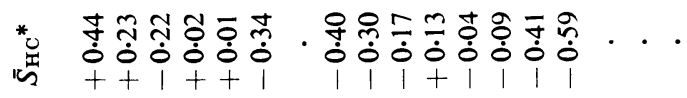

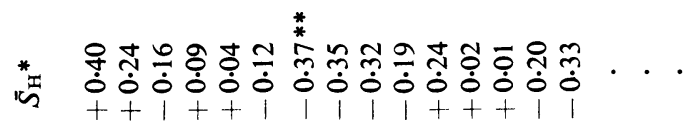

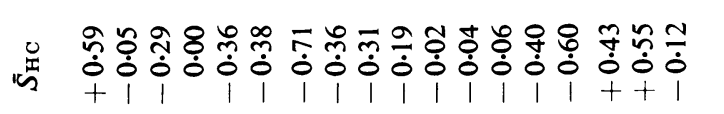







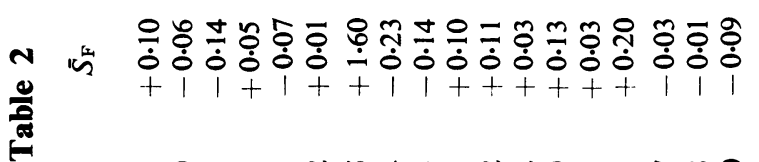

u

$\stackrel{\check{\widehat{\sigma}}}{\stackrel{\Xi}{\Xi}}$

$\bar{z}$

䄈

$\stackrel{\overline{0}}{\frac{0}{2}}$

䄱

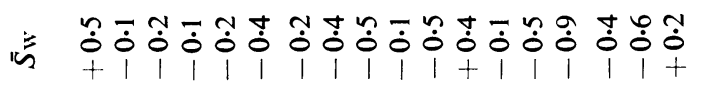

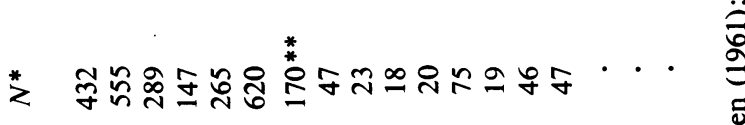

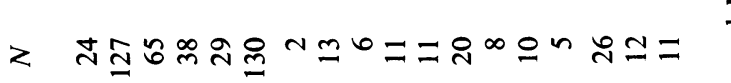







FIG. 2. Wake index $S_{W}$ as a function of semi-major axis and eccentricity; the distribution of $S_{W}$ in six regions of the diagram.

5 of 15 , or $33 \%$ of the meteors have $S_{\mathrm{w}}>1 \cdot 3$. This is to be compared with 16 cases of $S_{\mathrm{W}}>1.3$ among the remaining 398 meteors, i.e. $4 \%$. The distribution of wakes agrees with the finding of Jacchia, Verniani and Briggs (JVB, Table 20.3) that the wakes increase in brightness with decreasing geocentric velocity. Evidently, a significant correlation should be found also with the perihelion distance, since most bright wakes are found between $q=0.9$ and 1.0 . It is suggested, however, that a greater perihelion distance is not the primary factor in wake formation but only another feature characterizing meteors which enter the region of observability from the outer zone.

\subsection{BLENDING}

Blending is a directly observable result of the progressive dispersion of meteoritic matter along the trajectory. It appears as a gradual increase in length of the trail segments relative to the shutter breaks, and is also estimated in JVB using an arbitrary scale of 0 to 4 . The blending does not seem to be correlated with the mass or velocity of the meteor but depends on the angle of incidence. Denoting by $Z_{\mathrm{R}}$ the zenith distance 
of the radiant, the relation (JVB, Table 20.9) can be approximated by a simple empirical formula,

$$
S_{\mathrm{B}}=B+\cos Z_{\mathrm{R}}-1 \cdot 2 \text {. }
$$

The distribution of the blending index $S_{\mathrm{B}}$, shown in Figure 3, exhibits a marked maximum around the boundary between regions $A_{1}$ and $A_{2}$; there is practically no blending in regions $\mathrm{C}, \mathrm{E}$ and $\mathrm{F}$. All five meteors with $1.2<a<1.8$ and $0.4<e<0.7$ have $S_{\mathrm{B}}>2 \cdot 0$; such intensive blending is observed only in 15 of the remaining 408 meteors. Exceptional is the blending of the Draconid meteors.
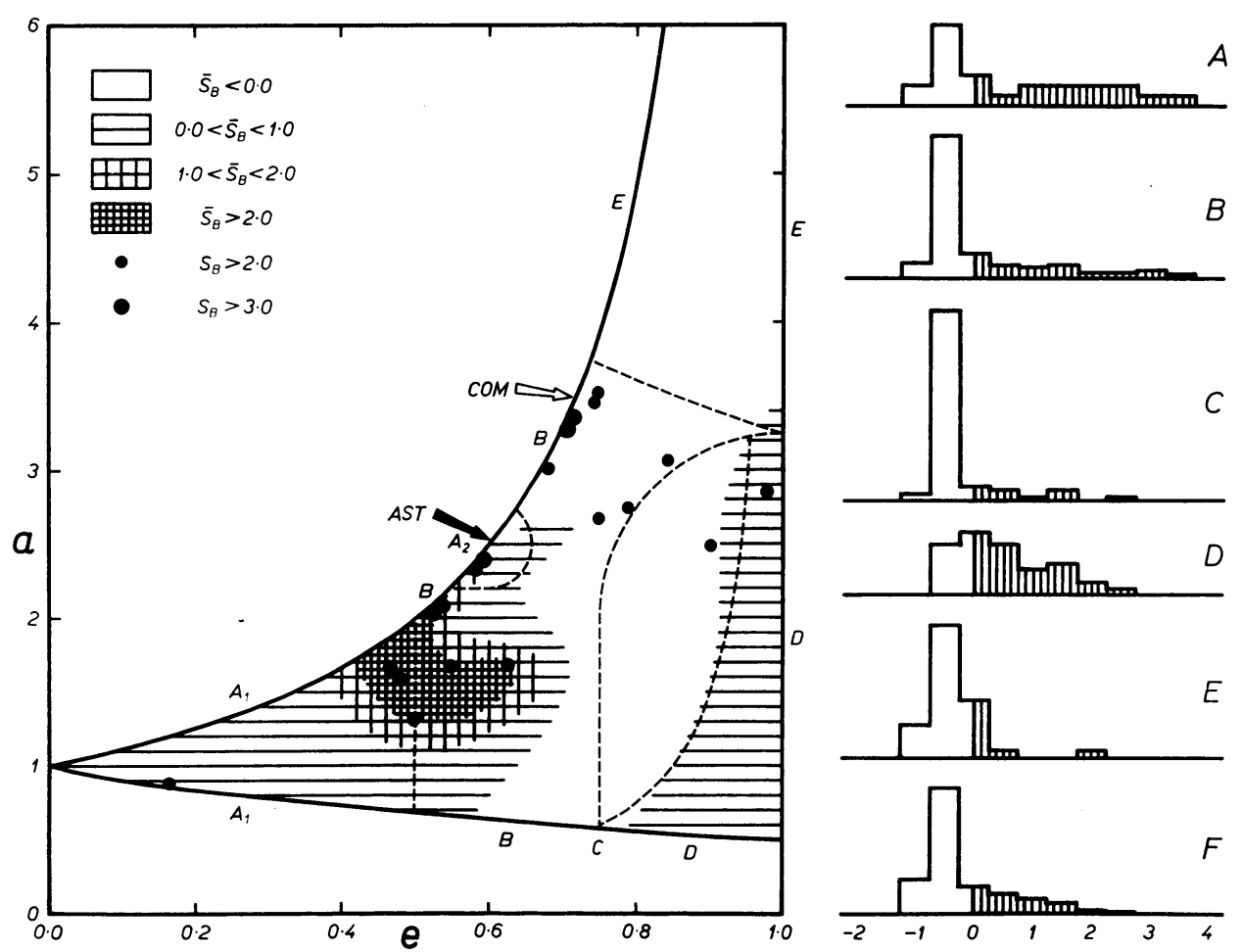

FIG. 3. Blending index $S_{B}$ as a function of semi-major axis and eccentricity; the distribution of $S_{B}$ in six regions of the diagram.

A strong terminal blending may perhaps mask, to some extent, the wake. Although there are meteors both with high wake index and high blending index (e.g. No. 8668 with $S_{\mathrm{W}}=1 \cdot 7, S_{\mathrm{B}}=2 \cdot 2$, No. 9880 with $S_{\mathrm{W}}=1 \cdot 5, S_{\mathrm{B}}=2 \cdot 1$ ), the value of the wake index may be underestimated in a few cases where the trail segments blend together. 


\subsection{COLOR INDEX}

The color index, defined as the difference between the photographic magnitude in the blue-sensitive Kodak X-ray emulsion and the magnitude estimate of a visual observer, was shown by Jacchia (1957) to be a function of the maximum photographic magnitude. In accordance with Table 15.2 of JVB we adopt

$$
\begin{array}{ll}
S_{\mathrm{C}}=C . I .+2 \cdot 0 & \text { for } \quad M_{\mathrm{pm}}<-2 \cdot 0, \\
S_{\mathrm{C}}=C . I .+1 \cdot 0+\left(1-M_{\mathrm{pm}}\right) / 3 & \text { for }-2 \cdot 0<M_{\mathrm{pm}}<1 \cdot 0, \\
S_{\mathrm{C}}=\text { C.I. }+1 \cdot 0 & \text { for } \quad M_{\mathrm{pm}}>1 \cdot 0 .
\end{array}
$$

At first glance it appears surprising that there is no significant dependence on velocity, in spite of the strong velocity dependence in the composition of meteor spectra. The reason is that the emission lines mostly contributing to the photographic magnitudes of fast and slow meteors ( $\mathrm{Ca}$ II doublet $\mathrm{H}$ and $\mathrm{K}$ and $\mathrm{Fe}$ I multiplets in the violet, respectively) fall approximately into the same spectral range. It appears that the low-excitation spectra of slow meteors are essentially composed of neutral iron


FIG. 4. Color index $S_{C}$ as a function of semi-major axis and eccentricity; the distribution of $S_{C}$ in six regions of the diagram 
lines, irrespective of the nature of the particle. Some excess towards the blue is indicated for meteors of shortest period, especially in region $A_{1}$. However, the mean difference is moderate, smaller than the dispersion of individual values due to the measuring errors. Hence the color index is unsuitable for discriminating among different types of meteors.

\subsection{PROGRESSIVE FRAGMENTATION}

While the blending is an observational evidence of meteor fragmentation, Jacchia's (1955) fragmentation index $\chi$ is a derived quantity, obtained by the comparison of the observed and theoretical deceleration and the mass-loss parameter. Also in this case the dependence on the angle of incidence is significant (JVB, Table 19.10) but its form is obviously different, due to a different scale. A decrease of the fragmentation index with increasing mass is clearly indicated when comparing the Super-Schmidt meteors with the small-camera meteors (JVB, Table 19.8) but it is observable only in the two extreme mass groups of the Super-Schmidt meteors, being practically absent within the range $0.05 \mathrm{~g}<m_{\infty}<5 \mathrm{~g}$ (JVB, Table 19.3). Therefore a simplified definition of the progressive fragmentation index $S_{\mathrm{F}}$ was applied, in the form

$$
S_{\mathrm{F}}=\chi+0.40 \cos Z_{\mathrm{R}}-0.53 \text {, }
$$

which fits well with the results of JVB, Table $19 \cdot 8$.

The distribution of the index $S_{\mathrm{F}}$ in the a/e diagram is shown in Figure 5 which, as expected, bears resemblance to Figure 3. The highest positive values of $S_{\mathrm{F}}$ are consistently concentrated in the part of region $B$ separating $A_{1}$ from $A_{2}$; next in regions $A$ and D. The two Draconids yield the highest and the third highest value of $S_{\mathrm{F}}$ of all 413 meteors. Highest negative values occur in region $\mathrm{C}$, which stands out very clearly when using this parameter. Extreme negative values of $S_{\mathrm{F}}$ characterize also the most abundant meteor shower of this region, the Southern Taurids. Comparison of the indices $S_{\mathrm{B}}$ and $S_{\mathrm{F}}$ (Figures 3 and 5, Table $2 ; S_{\mathrm{B}} \simeq 3 S_{\mathrm{F}}-0 \cdot 2$ ) yields a good agreement, supporting Jacchia's (1955) interpretation. Only the long-period showers of Perseids and Orionids give significantly higher $S_{F}$ than would be expected according to the blending. Since these meteors belong among those of smallest mass, the discordance may be due to the omission of the mass dependence of $S_{F}$ in Equation (7). However, the $\delta$ Aquarids also have small masses, and they show just the opposite deviation. The index of the Draconids is again quite exceptional.

\subsection{ABLATION}

The ablation coefficient $\sigma$ is obtained from the mass equation and luminosity equation, using measured values of brightness and deceleration. Jacchia, Verniani and Briggs list for each meteor the value which fits best along the whole trajectory. 

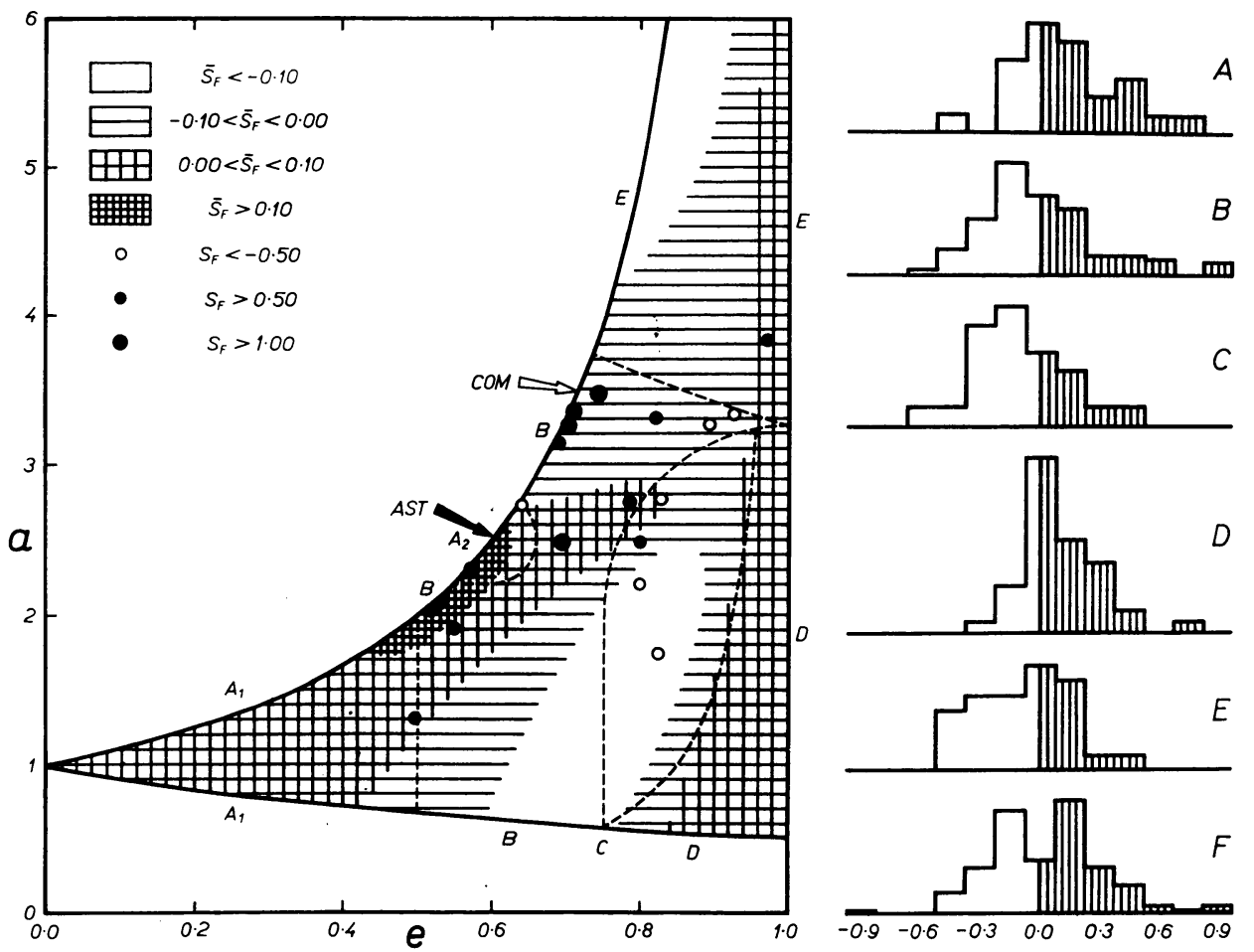

FIG. 5. Fragmentation index $S_{F}$ as a function of semi-major axis and eccentricity; the distribution of $S_{F}$ in six regions of the diagram.

There is a distinct dependence on velocity (JVB, Table $17 \cdot 1)$, which can be removed by a simple empirical correction, defining the ablation index $S_{\mathrm{A}}$ as

$$
S_{\mathrm{A}}=\log \sigma+0 \cdot 50 \log v_{\infty}+10 \cdot 40
$$

$\left(v_{\infty}\right.$ in $\mathrm{km} / \mathrm{sec}$ ). It must be noted that the velocity correction is a drawback to this procedure. Unlike the zenith angle or the apparent brightness, used for correction of other indices, the no-atmosphere velocity itself is a function of the position in the a/e diagram. When the velocity dependence is empirically removed from the data, the resulting distribution of $S_{\mathrm{A}}$ must be interpreted with caution.

This distribution is shown in Figure 6. The range of variation of $\bar{S}_{\mathrm{A}}$, the median, is small compared with the entire dispersion of individual values. Some excess in regions $A$ and $B$ as compared with $C$ and $D$ is indicated; however, an adjustment of the coefficient of $v_{\infty}$ in Equation (8) would cancel this difference. This is impossible for the region between $\mathrm{A}_{1}$ and $\mathrm{A}_{2}$ where negative indices $S_{\mathrm{A}}$ appear just at the same place where high values of the fragmentation and blending indices, $S_{\mathrm{F}}$ and $S_{\mathrm{B}}$, were found. The two Draconids are entirely exceptional, with a negative value of $S_{\mathrm{A}}$ 



FIG. 6. Ablation index $S_{A}$ as a function of semi-major axis and eccentricity; the distribution of $S_{A}$ in six regions of the diagram.

differing from the mean of other meteors by 27 times the median deviation; only two sporadic meteors, Nos. 7069 and 6961, differ from this by a factor of more than 5 . The apparently high positive $\bar{S}_{\mathrm{A}}$ for the Orionids is a median of three values only, one of them negative and two positive, and is therefore of no significance. It must be remembered that the intensity of ablation decreases with increasing $S_{\mathrm{A}}$.

\subsection{DECELERATION}

The deceleration index $S_{\mathrm{D}}$ was defined as a weighted mean of all measured differences,

$$
S_{\mathrm{D}}=\log \rho_{\mathrm{obs}}-\log \rho_{\mathrm{st}},
$$

where $\rho_{\mathrm{st}}$ is the density tabulated in the U.S. Standard Atmosphere (1962) for the observed height. JVB, Table $1 \cdot 2$ lists, in addition to this quantity, also the quantity $\log \rho_{\text {corr }}-\log \rho_{\text {st }}$, where a correction for the progressive fragmentation is applied. The distribution in the $a / e$ diagram was computed for both cases, but since there were no significant differences, the results are shown in Figure 7 for the observed values only. 

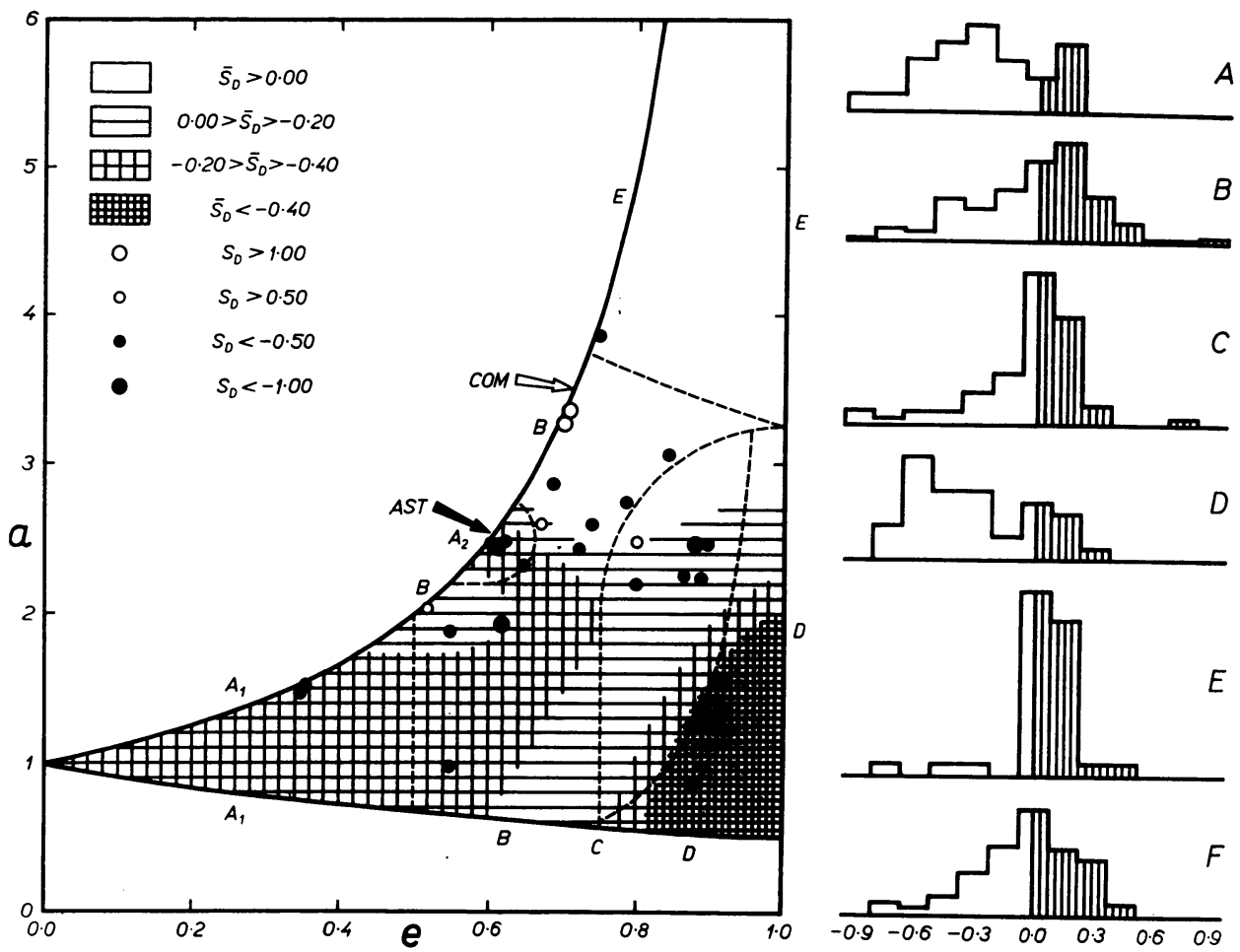

FIG. 7. Deceleration index $S_{D}$ as a function of semi-major axis and eccentricity; the distribution of $S_{D}$ in six regions of the diagram.

$\bar{S}_{\mathrm{D}}$ is slightly positive for all meteors of longer period and for the regions B and C. Higher negative values are found in regions $A$ and $D$, including the members of the Geminid stream but not those of the $\delta$ Aquarid stream. Exceptionally high positive values of $S_{\mathrm{D}}$ belong to the Draconids.

In this case the differences between individual regions of the $a / e$ diagram are more significant than in any other derived quantity. Even in this case the abundance histograms reveal no pure region that would not include meteors with the opposite sign of the index, nevertheless the displacements of the maxima in regions $A$ and $D$ to negative value of $S_{\mathrm{D}}$ stand out clearly.

\subsection{BEGINNING HEIGHTS}

A meaningful index $k_{\mathrm{b}}$, based on the measured beginning height of the luminous trajectory, has been derived by Ceplecha (1967). We shall denote as the height index $S_{\mathrm{H}}$ the quantity

$$
S_{\mathrm{H}}=k_{\mathrm{b}}-7 \cdot 20=\log \rho_{\mathrm{st}}+2 \cdot 50 \log v_{\infty}+0 \cdot 50 \log \cos Z_{\mathrm{R}}-7 \cdot 20 .
$$


As an alternative solution we apply a correction to the meteor mass based on the relation between mass and beginning height (JVB, Table $5 \cdot 1 \cdot 2$ ),

$$
S_{\mathrm{HC}}=S_{\mathrm{H}}-0 \cdot 20\left(\log m_{\infty}-\log m_{0}\right) \text {. }
$$

The reference mass $m_{0}$ is $0.2 \mathrm{~g}$ for the mass scale of Verniani (1964), and $0.031 \mathrm{~g}$ for the Öpik-Jacchia system used in previous listings, including the catalog of McCrosky and Posen (1961). Since the data needed for the computation of $S_{\mathrm{H}}$ are given in this catalog for a 6-times larger number of meteors than in JVB, we used them all for computing the median values in the a/e diagram and for constructing the abundance histograms. However, with regard to the lower accuracy of the reduction method, individual extreme values plotted in Figure 8 are taken only from the highprecision sample of Jacchia and Whipple (1961) used by Jacchia, Verniani and Briggs. A graphical representation is given only for $S_{\mathrm{H}}$, but there is a good agreement with $S_{\mathrm{HC}}$; the differences among the regions $\mathrm{A}-\mathrm{F}$ become even more pronounced when the mass correction is applied. This may be seen in Table 2, where both quantities are listed, separately for the data from JVB and for the complete catalog of McCrosky and Posen (denoted by an asterisk).

An enhanced number of meteors also permitted the construction of the abundance histograms of $S_{\mathrm{H}}$ for some major showers; these are shown in the lower part of Figure 8. The values of $S_{\mathrm{H}}$ for the Draconids were computed from the measurements of Jacchia et al. (1950). This means undoubtedly a loss on homogeneity with other data, in particular because the Draconid observations were made by small cameras of 45-75 $\mathrm{mm}$ focal length. Nevertheless the Draconids appear so extraordinary that they are worth comparing with the other showers even in spite of the lower accuracy.

The figures reflect the peculiar distribution of $k_{\mathrm{b}}$ pointed out by Ceplecha (1967). The highest values of $S_{\mathrm{H}}$ occur, with a conspicuously narrow dispersion, in regions $\mathrm{A}_{1}$ and $\mathrm{A}_{2}$; the lowest ones in regions $\mathrm{C}, \mathrm{E}$, and $\mathrm{F}$. The difference between the distribution of the meteors with $S_{\mathrm{H}}<-0.40$ and $S_{\mathrm{H}}>0.60$ in the a/e diagram is striking indeed, strongly supporting the usefulness of Ceplecha's parameter $k_{\mathrm{b}}$ for the classification of meteors. The broad dispersion of $S_{\mathrm{H}}$ in region B indicates a mixed character of its population, which is confirmed also by the distribution of the other indices.

An enhanced number of meteors in region $\mathrm{D}$ makes it possible to check that the height index $S_{\mathrm{H}}$ gradually increases with decreasing perihelion distance, the median value being +0.05 for meteors with $0.10<q<0.15$ and +0.30 for those with $q<0 \cdot 10$. This effect is also borne out by the $\delta$ Aquarids: 10 Aquarid meteors with $q>0.07$ yield $S_{\mathrm{H}}=+0.02$, while the other 10 , with $q<0.07$, yield $S_{\mathrm{H}}=+0.35$. In the Geminid shower the dispersion of perihelion distances is too small to make any difference observable.

The height indices of shower meteors in general agree well with the sporadic background in the respective areas of the diagram. The Draconids are quite exceptional, not only by the large negative value of $\bar{S}_{\mathrm{H}}$ but also by an abnormal dispersion of $S_{\mathrm{H}}$. 

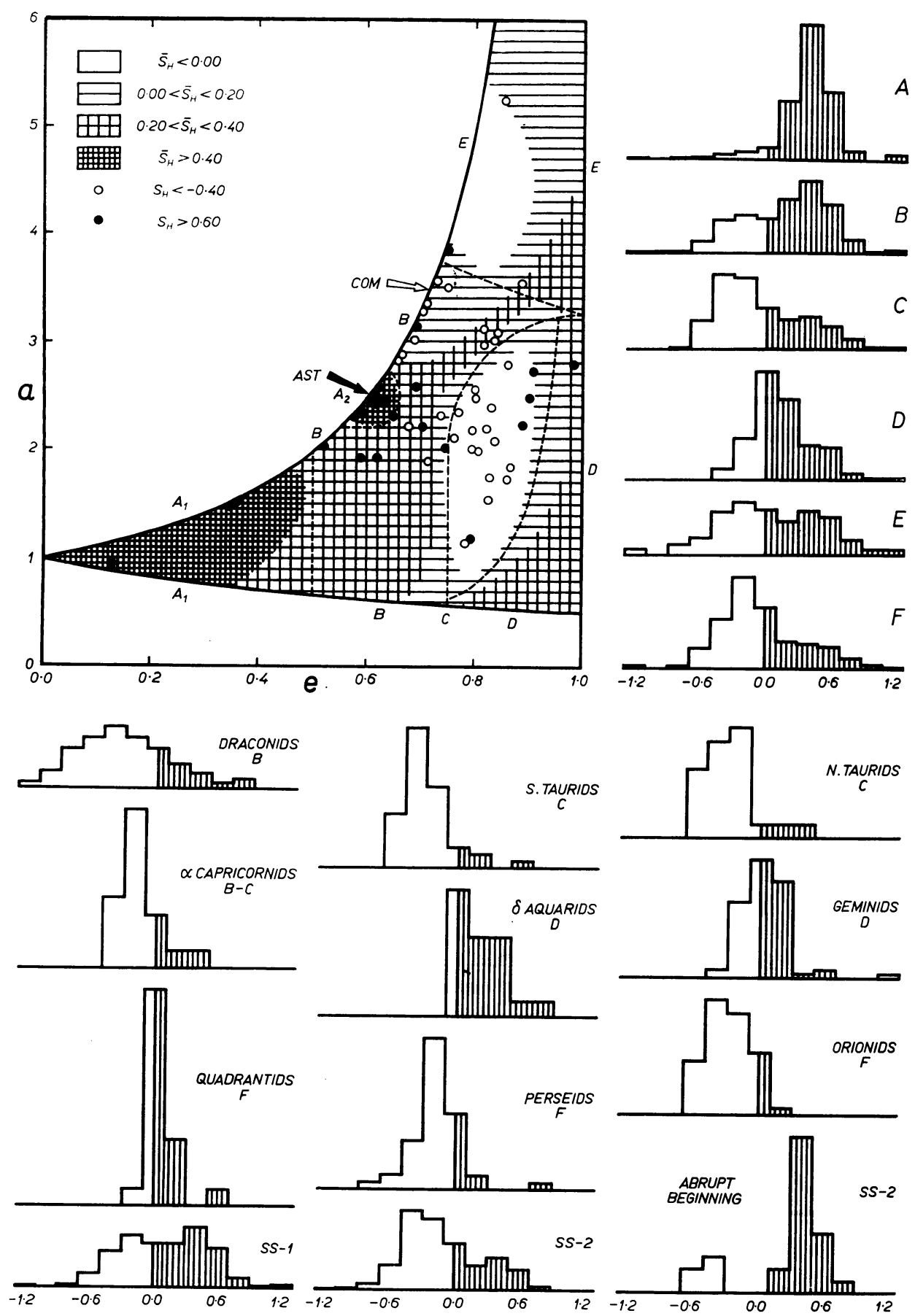

FIG. 8. Beginning height index $S_{H}$ as a function of semi-major axis and eccentricity; the distribution of $S_{H}$ in six regions of the diagram, in some major showers and in selected meteor samples. 
This is much too wide to be attributed to the lower precision of the data. It appears more likely that the Draconid stream includes a broad variety of meteoric material, as compared with the uniform Quadrantid, Capricornid or Perseid meteors, for example. The striking similarity of the two Draconids recorded by the Super-Schmidt cameras in 1953 cannot be simply extrapolated to the whole stream.

The sharp limitation of the Geminid and $\delta$ Aquarid distribution, and of the region $\mathrm{D}$ as a whole, on the side of negative height indices is consistent with the operation of a destructive effect having eliminated the less resistive meteoroids at repeated close approaches to the Sun. Such an explanation has already been suggested by Whipple (1963) for the physical peculiarities of the Geminids.

The last three histograms give the distribution in $S_{\mathrm{H}}$ for the complete SuperSchmidt material listed by McCrosky and Posen (1961, SS-1), for the high precision sample of Jacchia and Whipple (1961, SS-2), and for the 26 meteors of the latter list classified by Jacchia et al. (1967) as meteors with an abrupt beginning. The difference between SS-1 and SS-2 is due to the selection criteria for accurate reduction, which eliminate most low-velocity short-period meteors. The histogram for meteors with an abrupt beginning of the light curve shows a sharp separation into two groups with well-defined maxima, and a prevalent contribution of meteors with positive $S_{\mathrm{H}}$ to this type of meteor trajectory. Three of five meteors with negative $S_{\mathrm{H}}$ are Orionids.

The significant uniformity of individual values of $S_{\mathrm{H}}$, or $k_{\mathrm{b}}$, for just these meteors with an abrupt beginning, agrees with the suggestions of Ceplecha (1967). The small dispersion of $S_{\mathrm{H}}$ for the bona fide asteroidal meteors in region $\mathrm{A}_{1}$ and $\mathrm{A}_{2}$, as compared with the remainder of the data, suggests their better agreement with the simplifying assumptions on which the theoretical derivation of $k_{\mathrm{b}}$ is based.

\subsection{Peculiar trails}

Three kinds of peculiar trails are specially marked in the list of Jacchia, Verniani and Briggs. These are 26 trails with abrupt beginning of the light curve $(a), 12$ trails with unusually short duration, interpreted as broad flares $(s)$, and 11 trails with one or more fragments detached in the course of the trajectory $(f)$. The proportions of these three types of trails in percent, $P_{a}, P_{s}$ and $P_{f}$, respectively, in regions A-F and in different meteor streams are given in the last columns of Table 1.

The trails with abrupt beginning are relatively most numerous among shortperiod orbits, especially in regions $A_{1}$ and $A_{2}$. They are characterized by frequent blending and a negative deceleration index; further by the absence of wakes, a higher negative color index and high positive values of the height index. The three lastnamed features are common also to the short trails. Their large height indices $S_{\mathrm{H}}$ suggest that these are not simply flares on faint trajectories, which are otherwise below the plate limit. An irregular shape of these meteors seems to be a better ex- 
planation of the peculiarity. This type of meteor trail is quite uniformly distributed among the regions $\mathrm{A}-\mathrm{F}$.

The disruption of meteors into a few larger fragments (to be distinguished from the progressive fine fragmentation and blending) is very significantly correlated with meteor mass. It was detected in 8 of the 14 largest meteoroids of JVB (with $m_{\infty}>0.7 \mathrm{~g}$ in the Jacchia-Öpik system and $m_{\infty}>4.5 \mathrm{~g}$ in the Verniani system), but only in 3 of 399 meteoroids smaller than this limit. The relative frequency of this phenomenon in these two magnitude ranges is almost $80: 1$ ! Otherwise, the fragmenting meteoroids do not show any special characteristics. Most of them belong to the regions $B$ and $E$, just like most meteors of highest mass. This finding agrees with the expectation of Jones and Kaiser (1966) that stresses due to thermal shock would cause fracture of meteoroids only above a critical size limit. However, this size limit appears to be appreciably higher than expected by Jones and Kaiser. It cannot be excluded that similar fractures sometimes occur also in smaller bodies than those recorded in JVB, with the secondary fragments too faint to be detected.

\section{Conclusions}

It was shown that in the semi-major axis/eccentricity diagram of meteor orbits certain regions can be demarcated which yield meteors of specific physical characteristics. Differences between these characteristics are moderate, in general smaller than the internal dispersion of the data, but in the median values they appear rather significant.

For the problem of asteroidal origin of some meteors it is important that the two regions, $A_{1}$ and $A_{2}$, in which asteroidal meteors are expected according to their potential evolutionary paths, do show different characteristics from the other meteors, which are obviously associated with comets. These characteristics are low and conspicuously uniform beginning heights (reduced to a standard velocity and angle of incidence), high progressive fragmentation, low ablation, low deceleration, and bright wakes. A direct contrast to these characteristics is represented by meteors moving in short-period orbits of higher eccentricity and lower perihelion distance. Region C, which includes such orbits, is situated on both sides of the evolutionary path with the drag constant $C=a e^{-4 / 5}\left(1-e^{2}\right) \simeq 0 \cdot 7$, or $q_{0} \simeq 0 \cdot 35$. These meteors resemble those moving in long-period and high-inclined orbits (regions $E$ and F) except that they yield somewhat higher rate of ablation and lower rate of fragmentation. Regions $A_{1}$ and $A_{2}$ are separated from one another and from region $C$ by the transient region $B$, in which a wider variety of particles occur. Meteors with extremely short perihelion distances $(q<0 \cdot 15$, region $\mathrm{D})$ tend to resemble the bona fide asteroidal meteors by a progressive increase of fragmentation and decrease of reduced beginning heights and decelerations as the perihelion approaches the Sun.

The characteristics of shower meteors, investigated in detail by Millman (1967), 
agree fairly well with those of sporadic meteors located in the corresponding place of the diagram, i.e. with those moving in orbits of similar size and shape. For instance, the Taurid stream is a typical representative of region $\mathrm{C}$, and its two branches, the Northern and Southern Taurids, do not appreciably differ from one another in spite of the significant difference in magnitude distribution (Kresák, 1964). Quite extraordinary are the two Draconids recorded by Super-Schmidt cameras in 1953. However, the less accurate observations of 1946 (Jacchia et al., 1950) suggest that it would be unjustified to generalize this peculiarity with regard to the whole stream. It appears more likely that the Draconid stream includes a broad variety of meteor particles of different nature, which may be a characteristic feature of meteor streams of recent origin. As time goes on, the less resistive particles would become eliminated and the mean characteristics of the shower would approach those of the sporadic background in the corresponding region of the diagram. This explanation is supported by the meteors of extremely short perihelion distance (region D) including the major showers of Geminids and $\delta$ Aquarids. The distribution of height indices of these meteors is rather sharply cut off at the wing of less resistive particles, and the borderline appears to progress with decreasing perihelion distance.

This result is in broad agreement with Jacchia's (1963) explanation of the peculiarities of individual meteor showers using Whipple's (1951) theory of comets. The difference is that Whipple stresses the conditions of pressure under which the meteoroids were formed inside the comets, and attributes the principal distinctions among individual streams to the different natures of their parent comets. The problem of whether the peculiarities result from the origin of the stream or from its subsequent evolution, poses a question which can be answered by additional observations: Is the Draconid stream composed merely of the exceptional kind of particles represented by the two Harvard Super-Schmidt meteors, or are there also normal meteoroids that will constitute the stream after the elimination of the peculiar short-lived particles? And if so, what is the proportion? From this point of view, any future accurate observation of the Draconid meteors, at present prevented by an unfavourable position of their node, would be of utmost significance.

\section{Acknowledgements}

My sincere thanks are due to Mrs L. Durkovičová and Mrs L. Smíšková for their assistance in processing the extensive numerical data on which the present study is based.

\section{References}

Ceplecha, Z. (1967)

Jacchia, L.G. (1955)

Jacchia, L.G. (1957)

Jacchia, L.G. (1963)
Smithson. Contr. Astrophys., 11, 35.

Astrophys. J., 121, 521.

Astr. J., $62,358$.

in The Moon, Meteorites and Comets, ed. by B.M. Middlehurst and

G.P. Kuiper, University of Chicago Press, p. 774. 
Jacchia, L.G., Whipple, F. L. (1961) Smithson. Contr. Astrophys. 4, 97.

Jacchia, L. G., Kopal, Z., Millman, P. M. (1950) Astrophys. J., 111, 104.

Jacchia, L. G., Verniani, F., Briggs, R. E. (1967) Smithson. Contr. Astrophys., 10, 1.

Jones, J., Kaiser, T. R. (1966) Mon. Not. R. astr. Soc., 133, 411.

Kresák, L. (1964) Bull. astr. Inst. Csl., 15, 190.

Kresák, Ľ. (1967) Smithson. Contr. Astrophys., 11, 9.

McCrosky, R.E., Posen, A. (1961) Smithson. Contr. Astrophys., 4, 15.

Millman, P.M. (1967) Smithson. Contr. Astrophys., 11, 105.

U.S. Standard Atmosphere (1962) N.A.S.A., U.S.A.F. and U.S. Weather Bureau, Washington. Verniani, F. (1964) Smithson. Contr. Astrophys., 8, 141.

Whipple, F.L. (1951) Astrophys. J., 113, 464.

\section{DISCUSSION}

Ceplecha: Have you tried the long-period orbits with $a>6 \mathrm{AU}$ ?

Kresák: The long-period meteors are not included in the a/e diagrams, but they are included in the distribution histograms for region $\mathrm{E}$ (low inclination) and $\mathrm{F}$ (high inclination).

Halliday: The Canadian Super-Schmidts recorded one Draconid about 1953 which shows the same extreme characteristics as the two Harvard Super-Schmidt photographs.

McCrosky: A - 9th mag. Draconid meteor obtained by the Prairie Network has a beginning height of greater than $100 \mathrm{~km}$. This is some $15 \mathrm{~km}$ greater than any other object of comparable velocity. Thus, even for the more massive Draconids, the beginning heights define the origin almost as well as the orbit.

Shao: In the Harvard-Smithsonian Super-Schmidt meteor collection, there is at least one more unpublished Draconid meteor taken in 1958. The trajectory and orbital data show that this meteor is a Draconid member. The meteor trail also has an identical appearance of extreme fragmentation as those two reported by Jacchia. The beginning height, if I remember correctly, was $109 \mathrm{~km}$.

Levin: The proportion of meteors which obviously are divided into several parts, depends not only on the mass of the meteor body but also on the focal length of the camera used. For plates with better resolution there will be more meteors with obvious fragmentation.

Kresák: I agree with this. However, the frequency of visible fragmentation into two or more parts should be in this case increased also due to the larger masses of the meteors recorded by long-focus cameras. 